\title{
Comparison of methods for flow border detection in images of smoke visualization
}

\author{
Petr Caletka ${ }^{1, a}$, Ondrej Pech ${ }^{1}$, Jan Jedelsky $^{1}$, Frantisek Lizal $^{1}$ and Miroslav Jicha ${ }^{1}$ \\ ${ }^{1}$ Brno University of Technology, Faculty of Mechanical Engineering, Technicka 2896/2,616 69 Brno, Czech Republic
}

\begin{abstract}
A separation of the flow region from the surroundings is an essential step in the analysis of smoke visualization images. The separation can be performed using several detection methods from the image segmentation group. This paper deals with the border detection of the air flow downstream of a benchmark automotive vent using different threshold-based detection methods. An assessment of the methods on the basis of the resulting image quality is also addressed. The quality level depends on the quantity and brightness of disturbances in the background area. The disturbance is usually an isolated region of smoke, which naturally cannot be a part of the flow. Three representative images of different quality levels were selected for the detection, and three methods were used for the evaluation. Each of the methods was used to determine the threshold differently (by the level, by the ratio, and by the change of brightness). It is demonstrated that the change-based method with an appropriately selected parameter is the most convenient for images with the worst quality level while level- and ratio-based methods are only applicable for images of good quality.
\end{abstract}

\section{Introduction}

The smoke visualization is an experimental method used in fluid mechanics, especially for air flow studies. The main aim of the visualization is to get basic information about the flow field, e.g. its direction or shape, see Lezovic et al. [1]. One of the visualization outputs is the plane cut of the flow (of the image). There is a need to separate the flow area from the background by means of some segmentation technique. The flow border points are detected by the application of specific method of image segmentation, see Richter and Stastny [2].

Davies [3] divided techniques of image segmentation into three categories according to their principle (edgebased, region-based and thresholding). Spanel and Beran [4] described principles of the edge-based techniques and also global and local thresholding. McQuillan et al. [5] used the smoke visualization and the egde based technique for the indoor flow. Krska [6] visualized the flow downstream of the automotive vent by the smoke and then used the global thresholding for the border detection. Pech et al. [7] also visualized the flow downstream of the automotive benchmark vent using the smoke visualization, however the local thresholding was used for the border detection. Richter and Stastny [2] compared differences between the level-based (global thresholding) detection and the change-based (local thresholding) detection.

This paper deals with the detection of air flow shape from a benchmark automotive vent. This detection was done by thresholding in two planes perpendicular to each other. Three detection methods were used for the detection; each of them determines the threshold differently (by level, by ratio, and by change of brightness), see Richter and Stastny [2].

\section{Experimental setup}

According to Janotkova [8], the air flow behind the automotive benchmark vent at the volume rate $33.5 \mathrm{~m}^{3} \mathrm{~h}^{-1}$ has a character of the free isothermal turbulent jet (see Figure 1) with the Reynolds number of $10^{4}$ and the Archimedes number of $1.2 \times 10^{-3}$.

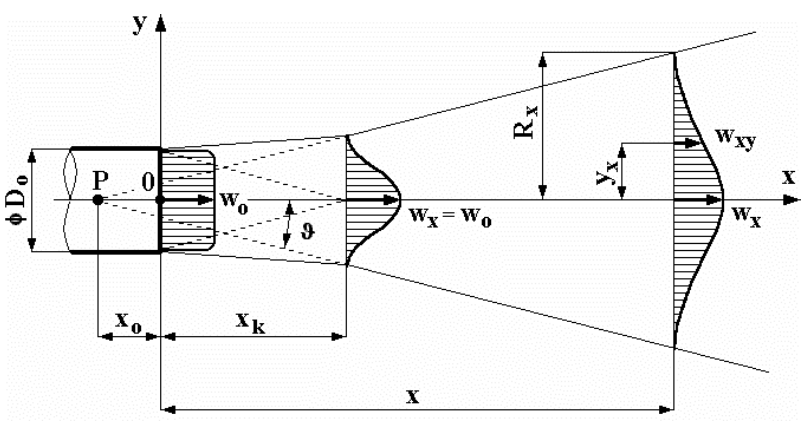

Figure 1. Typical shape of the free isothermal turbulent jet [8]

The jet was visualized by the smoke and illuminated with the use of a laser sheet. The quantity of the smoke fluid was represented by its consumption, which was set to $6 \mathrm{ml} \mathrm{min}{ }^{-1}$. The laser sheet output was set to $0.8 \mathrm{~W}$.

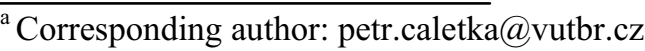


Horizontal and vertical planar cuts of the jet (see Figure 2) were taken with camera Canon EOS 300D with the lens Canon EF 17-40 mm f/4L USM. The EV value of the camera was set to 5.65. Setup of all of these components was documented by Pech et al. [7] during a complex optimization procedure.

\section{Evaluated images}

Each detection method was applied to a set of three images of different quality. The quality measure depends on the quantity and brightness of disturbed regions in the background area, see Figure 2 (white circles). The sensitivity to the disturbed regions is crucial for selection of the most appropriate method.
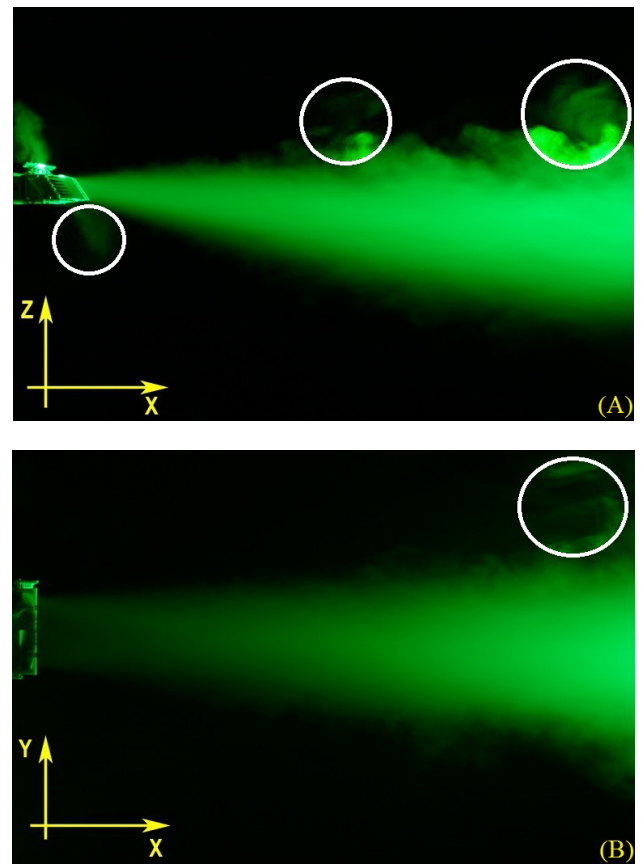

Figure 2. Disturbances in the images labelled by white circles: (A) side view; (B) top view

Caletka [9] assigned these images into three groups according to their quality:

a) with significant disturbed regions,

b) without significant disturbed regions,

c) without disturbed regions with computer retouching applied to background.

\section{Detection methods}

Richter and Stastny [2] documented that threshold-based methods for the jet border detection uses a number of cross-sections of the jet, which are perpendicular to the jet axis, see Figure 3. Each cross-section can be represented by the length $(l, \mathrm{x}$-axis) - brightness $(\mu, \mathrm{y}-$ axis) plot, see Figure 4.

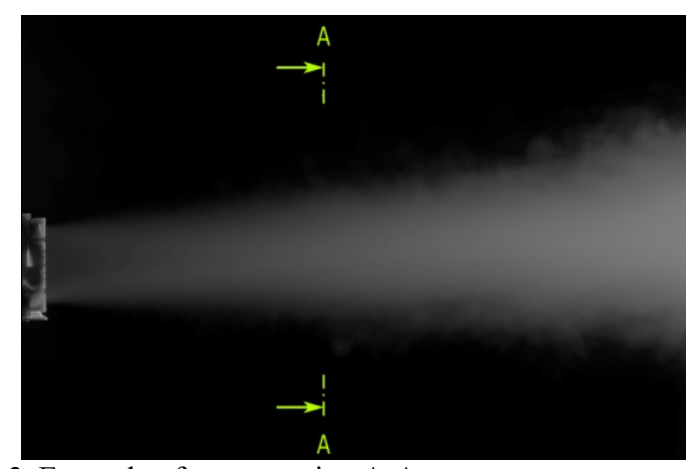

Figure 3. Example of cross-section A-A

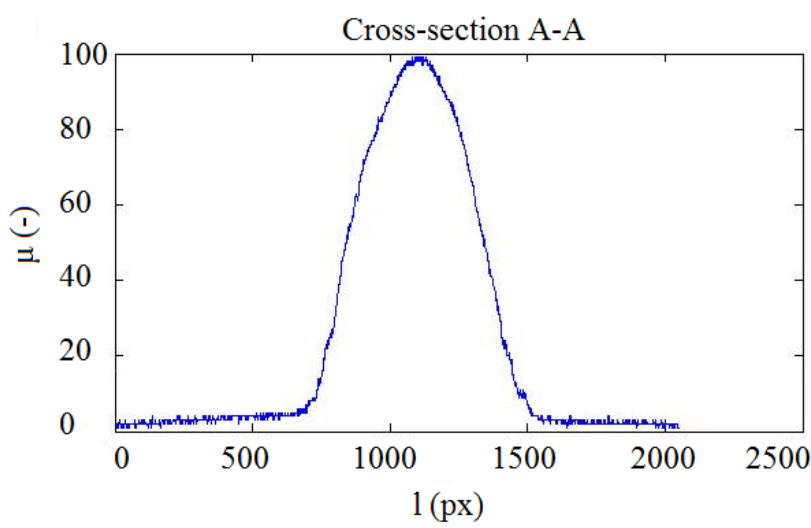

Figure 4. Length-brightness characteristic

A threshold $T(l, \mu)$, the border point of the jet, was determined in each cross-section by three different ways. First, the level-based detection from the global thresholding set of segmentation techniques was used. Then, two methods from the local thresholding set were used, namely the ratio- and change-based detection. The functionality and the character of each of the methods are controlled by adjustable parameters.

\subsection{Level-based detection}

Richter and Stastny [2] described a very simple principle of this detection method - global thresholding. There are border points $T$ indicated in each cross-section depending on the selected level of adjustable parameter $p_{L}$, see Figure 5. In this case, $p_{L}$ represents the jet border point, namely its brightness value $\mu$. The $p_{L}$ value was chosen as a specific percentile of the complete jet image brightness $\operatorname{maximum} \mu_{i, \max }$.

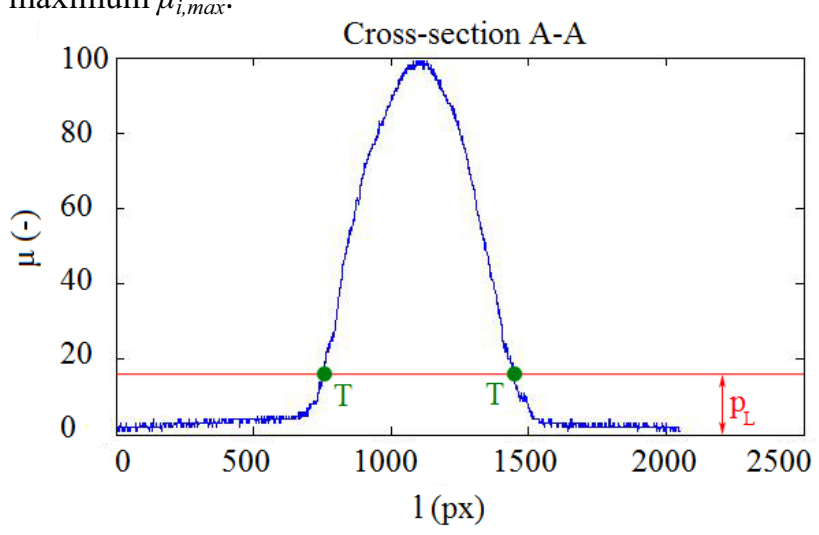

Figure 5. Schematic of the border detection in the level-based method 


\subsection{Ratio-based detection}

This detection method based on local thresholding was described by Caletka [9]. There are border points $T$ detected as the specific percentile $p_{R}$ of the each crosssection brightness maximum $\mu_{c, \max }$, see Figure 6 . One of advantages of the method is the elimination of the uneven lighting of the visualized jet.

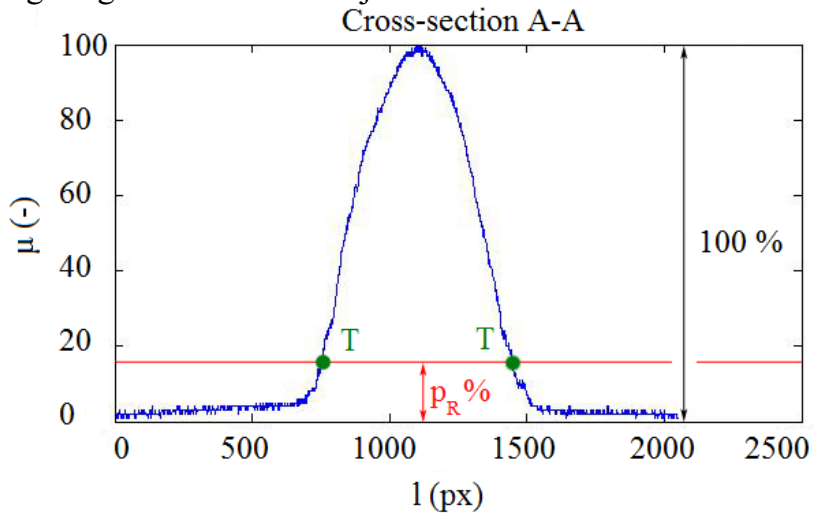

Figure 6. Schematic of the border detection in the ratio-based method

\subsection{Change-based detection}

This detection method belongs to the group of local thresholding methods and its principle (see Figure 7) is more complex than in case of the previous methods.

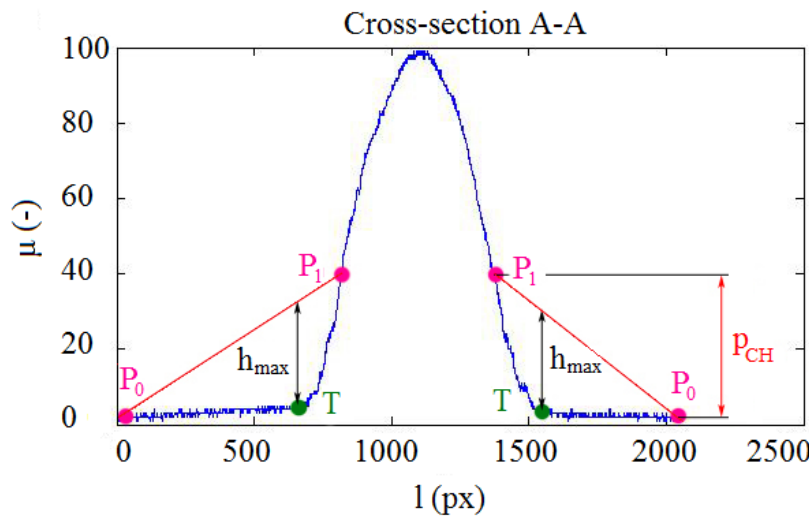

Figure 7. Schematic of the border detection in the change-based method

The extreme points $p_{0}$ of each cross-section are automatically assigned to the background, as documented by Richter and Stastny [2]. The points $P_{I}$ have the same brightness value $\mu$ as the chosen adjustable change parameter $p_{C H}$. There is also a presumption that these points are assigned to the flow area. The $p_{C H}$ value is always selected as a specific percentile of brightness minimum in the axis of flow $\mu_{a, \min }$. As can be seen in Figure 7, the height $h$ between the segment line $\left|p_{0} p_{l}\right|$ and the current $\mu$ is traced. When the perpendicular line $h_{\max }$ is found, the point with current $\mu$ is indicated as the border point $T$.

\section{Results and discussion}

The approximate shape of the jet can be predicted on the basis of theory of free isothermal turbulent jet, see Janotkova [8] and Section 2. However, Seda [10] also studied and measured the jet properties, especially its velocity field by the CTA (constant temperature anemometry). The jet shape detected from his CTA measurement (see Figure 8) corresponds with the assumption presented in Janotkova [8].

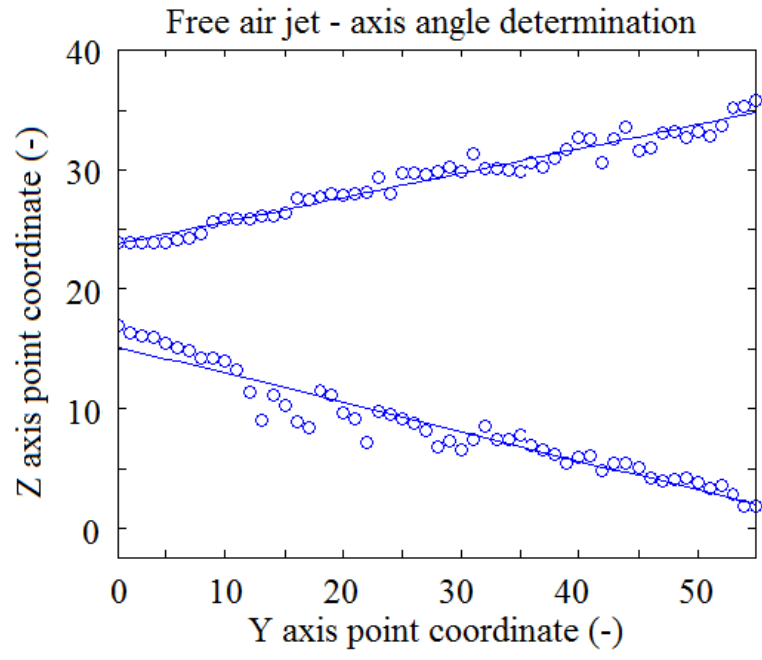

Figure 8. The jet shape indicated by CTA measurement [10]

A suitable detection method for jet border detection should be designed in order to not interpret the disturbed regions as a jet area. All the detected jet shapes in bottom-view images (X-Y plane) are presented in Figures 9-11. The specific values of adjustable parameters used in this work were selected as described in Caletka [9].

\subsection{Image with significant disturbed regions}

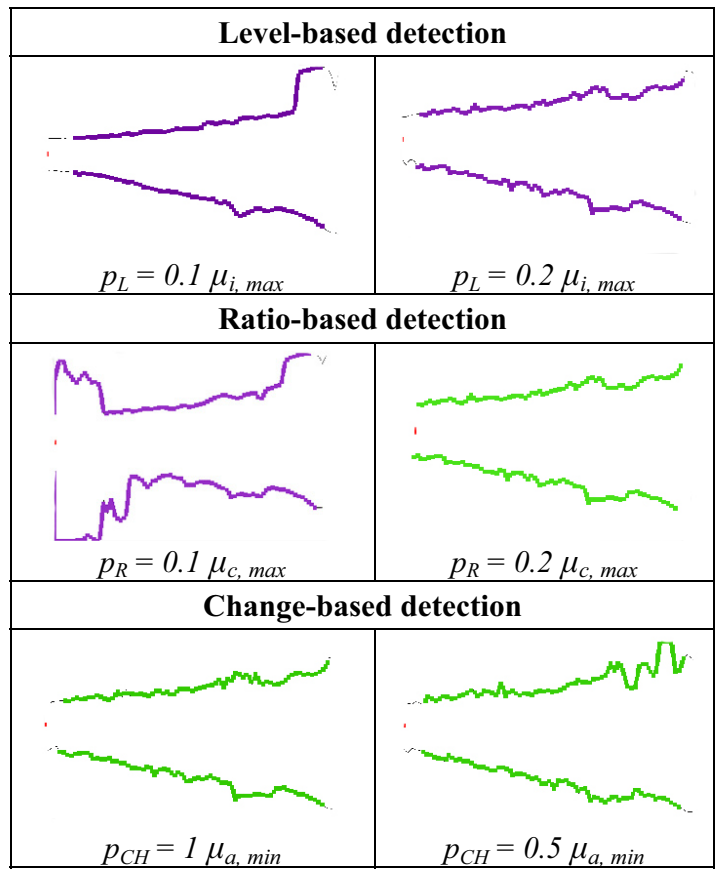

Figure 9. Jet shapes detected from the image with significant disturbed regions by all the methods 


\subsection{Image without significant disturbed regions}

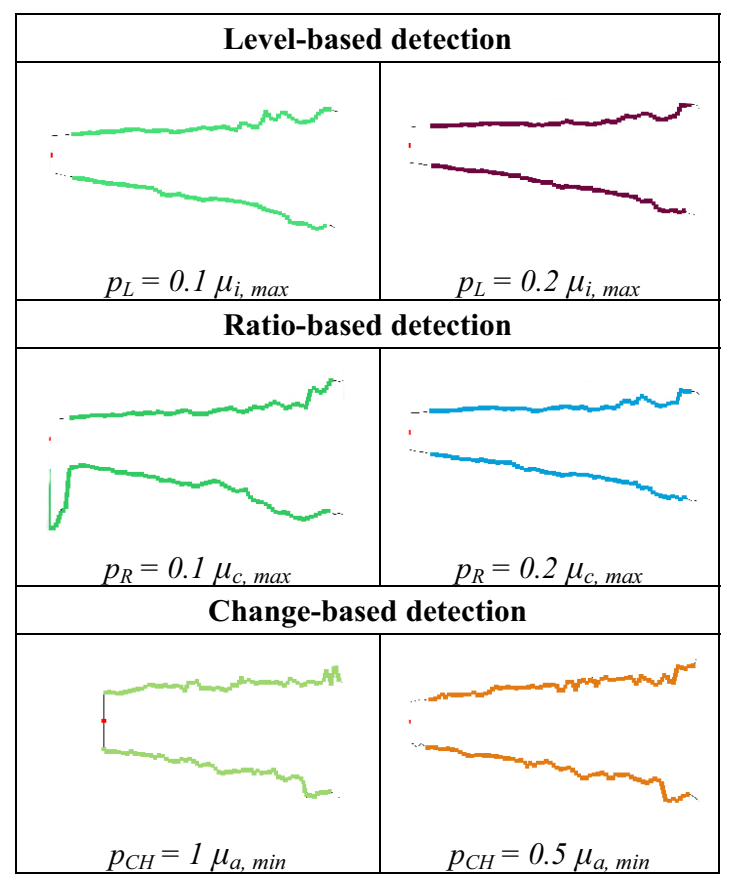

Figure 10. Jet shapes detected from the image without significant disturbed regions by all the methods

\subsection{Image without the disturbed regions}

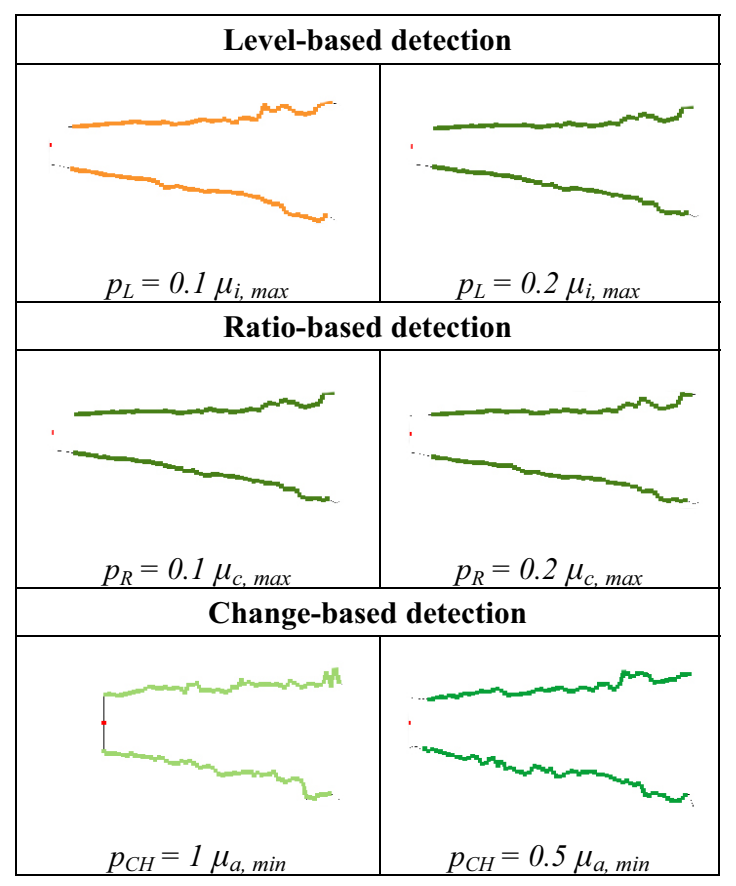

Figure 11. Jet shapes detected from the image without the disturbed regions by all the methods

\section{Conclusions}

It was found that the level-based method detection with a lower value of $p_{L}$ is suitable especially in images without significant disturbed regions. When the quantity of disturbed regions increases it is possible to eliminate this issue by a higher value of $p_{L}$. However, this procedure is not optimal for correction of the border detection owing to the global character of this threshold method.

In the case of the ratio-based detection, it was found that the lower value of $p_{R}$ is only suitable for images without the disturbed regions. The detection with the lower value of $p_{R}$ is very sensitive to the disturbed regions, so when their quantity increases, the method does not work correctly. However, this issue can be eliminated by higher value of $p_{R}$. This procedure is the correct way to get relevant results due to the local character of this threshold method.

Finally, in case of the change-based detection, it was found that the lower value of $p_{C H}$ is suitable for images with diverse quality levels. With a higher value of $p_{C H}$, the sensitivity to the presence of disturbed regions decreases. On the other hand there is a higher risk of wrong detection of jet shape immediately at the outlet of the benchmark automotive vent. The change-based method with an appropriately chosen parameter $p_{C H}$ is the most universal. Flow borders detected using this method were not influenced by disturbed regions and this method was therefore found suitable for further application. Methods that find the border points according to the level or the ratio are suitable only for images with better quality, and in such cases usually with the $p_{L}$ or $p_{R}$ higher than 20 .

\section{Acknowledgements}

The presented research was supported by the project LO1202 NETME CENTRE PLUS with the financial support from the Ministry of Education, Youth and Sports of the Czech Republic under the "National Sustainability Programme I" and by the project reg. number FSI-S-14-2355 of the Brno University of Technology. The authors also gratefully acknowledge the support from the "Josef Bozek Competence Centre for Automotive Industry", number TE01020020 of the Technology agency of the Czech Republic.

\section{References}

1. T. Lezovic, F. Lizal, J. Jedelsky, M. Jicha, HVAC\&R Research, 19, 9 (2013)

2. J. Richter, J. Stastny, Advances in Computer Science, 6, (2012)

3. E. R. Davies, Computer \& Machine Vision: Theory, Algorithms, Practicalities, 4, 912 (2012)

4. M. Spanel, V. Beran, Obrazové segmentační techniky prehled existujicich metod, Brno University of Technology; (2006)

5. B. McQuillan, J. Herzberg, L. D. Montoya, B\&E 73, 9 (2014)

6. L. Krska, Master Diploma Thesis, Brno University of Technology, 82, (2011)

7. O. Pech, J. Jedelsky, P. Caletka, M. Jicha, EPJ Web of Conferences, 67, 4 (2014)

8. E. Janotkova, Technika prostředí 1. část, 58 (2011)

9. P. Caletka, Bachelor Thesis, Brno University of Technology, 66, (2014)

10. L. Seda, Master Diploma Thesis, Brno University of Technology, 72, (2015) 